\title{
Article
}

\section{Rubrene: The interplay between intramolecular and intermolecular interactions determines the planarization of its tetracene core in the solid state}

\author{
Christopher Sutton, Michael S. Marshall, C. David Sherrill, Chad Risko, and Jean-Luc Bredas
}

J. Am. Chem. Soc., Just Accepted Manuscript • DOI: 10.1021/jacs.5b04066 • Publication Date (Web): 15 Jun 2015

Downloaded from http://pubs.acs.org on June 21, 2015

\section{Just Accepted}

"Just Accepted" manuscripts have been peer-reviewed and accepted for publication. They are posted online prior to technical editing, formatting for publication and author proofing. The American Chemical Society provides "Just Accepted" as a free service to the research community to expedite the dissemination of scientific material as soon as possible after acceptance. "Just Accepted" manuscripts appear in full in PDF format accompanied by an HTML abstract. "Just Accepted" manuscripts have been fully peer reviewed, but should not be considered the official version of record. They are accessible to all readers and citable by the Digital Object Identifier (DOI®). "Just Accepted" is an optional service offered to authors. Therefore, the "Just Accepted" Web site may not include all articles that will be published in the journal. After a manuscript is technically edited and formatted, it will be removed from the "Just Accepted" Web site and published as an ASAP article. Note that technical editing may introduce minor changes to the manuscript text and/or graphics which could affect content, and all legal disclaimers and ethical guidelines that apply to the journal pertain. ACS cannot be held responsible for errors or consequences arising from the use of information contained in these "Just Accepted" manuscripts. 


\title{
Rubrene: The interplay between intramolecular and intermolecular interactions determines the planarization of its tetracene core in the solid state
}

\author{
Christopher Sutton, ${ }^{1}$ Michael S. Marshall, ${ }^{1}$ C. David Sherrill, ${ }^{1}$ \\ Chad Risko, ${ }^{1,2, *}$ and Jean-Luc Brédas ${ }^{1,3, *}$ \\ ${ }^{1}$ School of Chemistry and Biochemistry \\ and Center for Organic Photonics and Electronics \\ Georgia Institute of Technology \\ Atlanta, Georgia 30332-0400 USA \\ ${ }^{2}$ Department of Chemistry \\ and Center for Applied Energy Research \\ University of Kentucky \\ Lexington, Kentucky 40506-0055 USA \\ ${ }^{3}$ Solar \& Photovoltaics Engineering Research Center \\ Physical Science and Engineering Division \\ King Abdullah University of Science and Technology \\ Thuwal 23955-6900, Kingdom of Saudi Arabia
}

\footnotetext{
*Email: chad.risko@uky.edu; jean-luc.bredas@,kaust.edu.sa
} 


\begin{abstract}
.
Rubrene is one of the most studied molecular semiconductors; its chemical structure consists of a tetracene backbone with four phenyl rings appended to the two central fused rings. Derivatization of these phenyl rings can lead to two very different solid-state molecular conformations and packings: One in which the tetracene core is planar and there exists substantive overlap among neighboring $\pi$-conjugated backbones; and another where the tetracene core is twisted and the overlap of neighboring $\pi$-conjugated backbones is completely disrupted. State-of-the-art electronic-structure calculations show for all isolated rubrene derivatives that the twisted conformation is more favorable (by -1.7 to $-4.1 \mathrm{kcal} \mathrm{mol}^{-1}$ ), which is a consequence of energetically unfavorable exchange-repulsion interactions among the phenyl side groups. Calculations based on available crystallographic structures reveal that planar conformations of the tetracene core in the solid state result from intermolecular interactions that can be tuned through well-chosen functionalization of the phenyl side groups, and lead to improved intermolecular electronic couplings. Understanding the interplay of these intramolecular and intermolecular interactions provides insight into how to chemically modify rubrene and similar molecular semiconductors to improve the intrinsic materials electronic properties.
\end{abstract}




\section{Introduction.}

A great challenge in the design of organic molecular materials (crystals) for electronics applications is that the intermolecular interactions that define the solid-state structure consist of weak van der Waals forces. Hence, external factors can readily influence molecular packing, with effects ranging from (random) small molecular shifts along different lattice directions to complete alterations of the crystal packing motif (polymorphism). ${ }^{1-3}$ Importantly, polymorphs can display markedly different electronic properties $^{3}$ simply due to the variations in molecular packing. ${ }^{4,5}$ While the solid-state conformation and packing of organic molecules intimately depends on the growth conditions, the chemical structure plays an obvious, defining role. ${ }^{6-9}$

A classic example is tetracene functionalized with phenyl rings at the 5-, 6-, 11-, and 12positions, a compound referred to as rubrene, 1 (see Figure 1). The presence of these phenyl rings converts the typical herringbone structure found in oligoacenes to a slipped-cofacial packing of the $\pi$-conjugated tetracene backbones. For rubrene, large hole mobilities (as high as $40 \mathrm{~cm}^{2} \mathrm{~V}^{-1} \mathrm{~s}^{-1}$ ) ${ }^{10}$ arise from the strong intermolecular electronic couplings (on the order of 100 $\mathrm{meV}$, as calculated with density functional theory methods) ${ }^{11}$ that result from the good wavefunction overlap among the frontier molecular orbitals of the stacked molecular neighbors in the crystallographic $a b$-plane of the orthorhombic crystal. ${ }^{12,13,14,15,16}$ However, electronic-structure calculations on isolated rubrene molecules show that the presence of the side phenyl rings makes the tetracene backbone of rubrene preferentially twist $\left(\sim 40^{\circ}\right),{ }^{17-20}$ which is confirmed by experimental evidence of twisted conformations both in solution ${ }^{18,21}$ and thin films. ${ }^{17,22}$ Interestingly, examination of rubrene thin films grown on $\mathrm{Au}(111)$ surfaces reveals that the twisted conformation is present in the initial layers and then transitions solely to the planar 
conformation, an indication of the decisive influence that surrounding molecules play in leading to a planar conformation in the bulk molecular structure. ${ }^{17}$

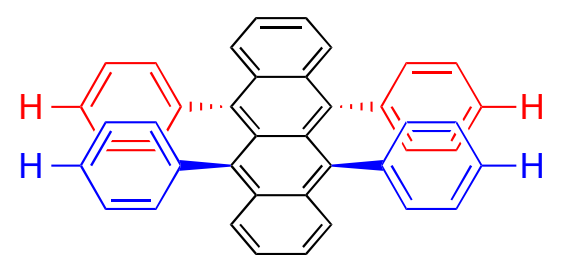

1

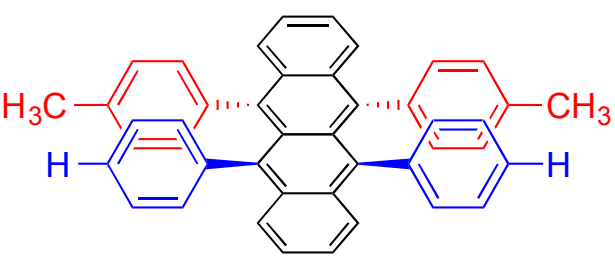

2

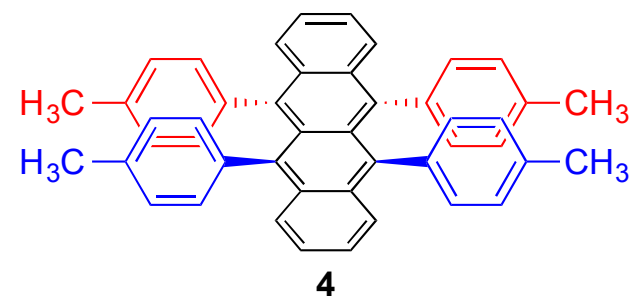

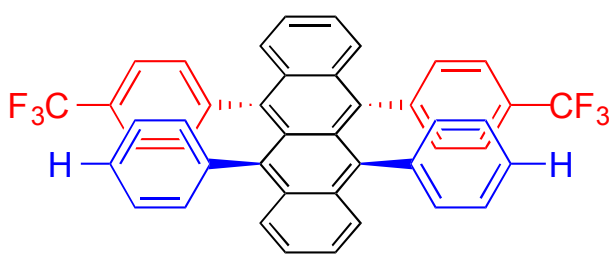

3

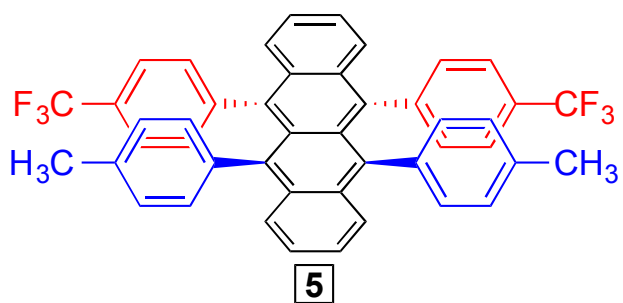

Figure 1. Chemical structures of some of the representative rubrene derivatives $-\mathbf{1}^{23}$ and $\mathbf{2}-\mathbf{5}^{19}-$ investigated in this study. $\mathbf{1}, \mathbf{3}$, and $\mathbf{5}$ display planar tetracene backbones in the crystal structure (indicated with boxes), while $\mathbf{2}$ and $\mathbf{4}$ are twisted. A complete list of all investigated 13 rubrene derivatives is provided in Figure S1 of the Supporting Information (SI).

It is important to underline that the twisted rubrene conformation is not exclusively found in isolated molecules and disordered solid-state materials; indeed, crystals of numerous rubrene derivatives, where the peripheral phenyl rings are substituted with, for example, alkyl, alkoxy, or fluorinated groups, indicate a seemingly random choice between twisted and planar conformations. ${ }^{21,24-26}$ Examples of this conformational variation are shown in Figure 1 where crystals of $\mathbf{1}, \mathbf{3}$, and $\mathbf{5}$ have planar tetracene cores, while in crystals of $\mathbf{2}$ and $\mathbf{4}$ the tetracene 
backbones substantially twist. Rubrene derivatives that maintain planar tetracene backbones can have intermolecular electronic couplings that even surpass that of the parent compound $\mathbf{1}$ (though their charge-carrier mobilities in single-crystal field-effect transistors currently remain below those for $\mathbf{1}) .^{24}$

For these reasons, rubrene and its derivatives provide a distinctive platform to study how chemical modification affects the competition between preferred molecular conformation and crystal packing. Here, we use a combination of density functional theory (DFT) and symmetryadapted perturbation theory $(\mathrm{SAPT})^{27}$ to investigate the non-covalent interactions at play in rubrene derivatives. Our goal is to gain a fundamental understanding of the factors that govern the molecular and packing structures in the solid state, in order ultimately to provide a basis for the development of new rubrene-based materials. Our study focuses in particular on how intramolecular and intermolecular interactions - understood in terms of exchange-repulsion, induction, dispersion, and electrostatics (with each of these terms discussed in detail in the Supporting Information, SI) - affect the planarity of the rubrene backbone. We begin by examining the propensity of the tetracene backbone to twist in isolated molecules and then show how this tendency to twist can be overcome in the solid state through interactions with molecular neighbors. Implications for functionalization strategies to control bulk systems are then derived and generalized through a comparison with the oligoacene series. We also discuss the explicit relationship between repulsive exchange interactions and intermolecular electronic couplings of $\pi$-stacked materials, and how conjugated structures can be chemically modulated to bring them into closer contact. 


\section{Methodology.}

DFT analyses of the neutral ground states were carried out using a variety of density functionals containing empirically parameterized dispersion interactions that were found to perform well in a previous benchmark study: ${ }^{28}$ B3LYP and B3LYP-D, ${ }^{23-25}$ IP-tuned $\omega$ B97 and $\omega$ B97-D, ${ }^{29}$ and the M05-2X $\mathrm{X}^{30}$ functional. The choice of functional, including the inclusion of dispersion corrections, has little effect on the results (see Tables S1 and S2 in the SI). All geometry optimizations were performed with the cc-pVDZ basis set. ${ }^{31}$ Frequency analyses were performed for the optimized geometries to ensure that a minimum had been reached. Transfer integrals, $t_{A B}$, for molecular dimers selected from the crystal structures were evaluated using a fragment orbital approach in combination with a basis set orthogonalization procedure at the B3LYP/cc-pVDZ level. ${ }^{32,33}$

For the long-range corrected hybrid $\omega \mathrm{B} 97$ functional, ${ }^{29}$ the optimal range-separation parameter $\omega$ (i.e., the $\omega$ value minimizing the many-electron self-interaction error) was determined following a non-empirical IP-tuning procedure ${ }^{34}$ where the difference between the highest occupied molecular orbital (HOMO) eigenvalue and the computed vertical ionization potential was minimized through the relation:

$$
J(\omega)=-\varepsilon_{H O M O}^{\omega}-\left(E_{g S}(\omega, N)-E_{g S}(\omega, N-1)\right)
$$

where $\varepsilon_{\text {HOMO }}^{\omega}$ is the HOMO eigenvalue of the ground state and $E_{g s}(\omega, N)$ and $E_{g s}(\omega, N-1)$ are the closed-shell neutral and radical-cation ground-state energies, respectively.

In order to evaluate the non-covalent interactions in terms of their exchange, induction/polarization, electrostatic, and London dispersion components, symmetry-adapted 
perturbation theory $(\mathrm{SAPT})^{27}$ with density fitting, to allow for efficient wave-function-based computations, was employed. ${ }^{35-37}$ We considered the so-called SAPT0 approximation, which does not include the effect of intramonomer electron correlation and therefore reduces the cost of this approach for studies on systems of large size, such as the rubrene derivatives. ${ }^{27,36} \mathrm{We}$ used a truncated aug-cc-pVDZ basis set ${ }^{31}$ that neglects diffuse functions on $\mathrm{H}$ atoms and diffuse $d$ functions on other atoms (so-called jun-cc-pVDZ ${ }^{38}$ ). Previously, the SAPT0/jun-cc-pVDZ approach was shown to give accurate stacking energies in non-covalent systems ${ }^{35,36}$ and performed well for the S22 test set of non-covalent interactions, ${ }^{36,39}$ for which higher-quality benchmark data are available. $^{40}$

\section{Results and Discussion. \\ Isolated Molecules.}

For the isolated rubrene derivatives, we report the total energies of the fully-relaxed (i.e., twisted tetracene backbone) and constrained-planar (i.e., the tetracene backbone is forced to maintain planarity) geometries evaluated via DFT calculations with IP-tuned $\omega$ B97. Results for the entire series of functionalized rubrene derivatives are provided in Table S1 of the SI. Across the series, the rubrene conformation with the twisted tetracene core $[\Delta \mathrm{E}=\mathrm{E}$ (twisted) $-\mathrm{E}($ planar $)]$ is favored by ca. -2 to $-4 \mathrm{kcal} \mathrm{mol}^{-1}$ over the constrained-planar tetracene backbone, a result consistent with previous theoretical studies. ${ }^{17-21}$ The degree of twisting, defined as $\theta_{\mathrm{B}}$ in Figure 2 , falls between 30 to $40^{\circ}$. The nearly equivalent energy differences across the series between the (constrained) planar and (relaxed) twisted backbones indicate that the exact nature and positions of the substituents on the phenyl rings seem to have only a small effect on the energetics of the 
molecular structure. We note for an isolated tetracene molecule that the planar conformation is some $5 \mathrm{kcal} \mathrm{mol}^{-1}$ (Table S5) more stable than conformations with twists similar to those in rubrene; ${ }^{41}$ hence, the tetracene backbone finds itself in a highly unfavorable conformation in rubrene.

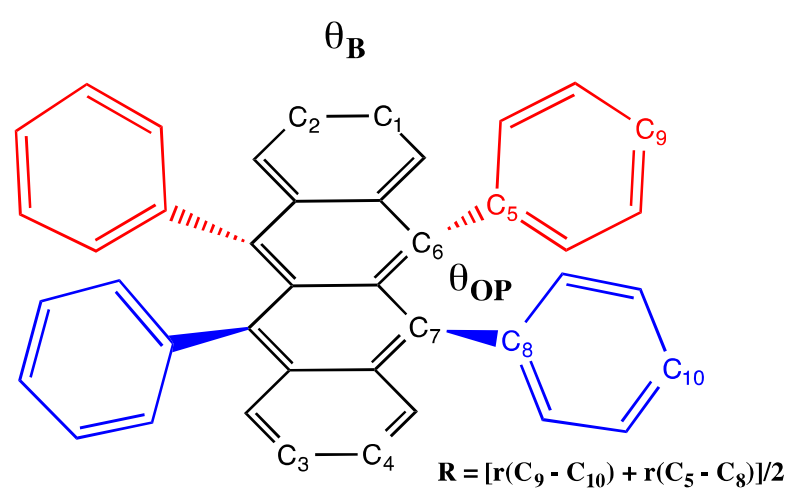

Figure 2. Drawing depicting the defined backbone $\left[\theta_{B}=\left(C_{1}-C_{2}-C_{3}-C_{4}\right)\right]$ and out-of-plane phenyl $\left[\theta_{\mathrm{OP}}=\left(\mathrm{C}_{5}-\mathrm{C}_{6}-\mathrm{C}_{7}-\mathrm{C}_{8}\right)\right]$ torsions in the rubrene systems under investigation. The definition of the separation between phenyl rings, given by the average distance $\mathrm{R}$ between the two carbons in the para positions $\left(\mathrm{C}_{9}\right.$ and $\left.\mathrm{C}_{10}\right)$ and the two carbons directly connected to the tetracene backbone $\left(\mathrm{C}_{5}\right.$ and $\mathrm{C}_{8}$ ), is also provided.

To detail the physical mechanisms at play that enforce the higher-energy twisted tetracene conformation, we focus on molecules 1 - 5 (Figure 1), which are representative of the entire series. We note that $\mathbf{2}$ and $\mathbf{4}$ are substituted solely with electron-donating methyl groups, while $\mathbf{3}$ and $\mathbf{5}$ are analogous molecules where one pair (syn-arranged) of methyl groups is replaced with perfluoromethyl groups. As noted above, these molecular pairs illuminate critical differences in terms of the molecular conformations found for isolated and solid-state rubrenes: ${ }^{19}$ While all of these molecules have similar (twisted) isolated-molecule structures, $\mathbf{2}$ and $\mathbf{4}$ maintain the twisted tetracene conformation in the crystal while the tetracene backbones in $\mathbf{3}$ and $\mathbf{5}$ take on a planar conformation similar to that of the parent compound 1. At the molecular level, one might expect 
a priori increased steric interactions in $\mathbf{4}$ and $\mathbf{5}$ and more distorted structures when compared to $\mathbf{2}$ and 3, as the former are para-substituted on all four phenylene rings. However, neither the degree of twisting nor the energy differences between the planar and twisted conformations follow this expectation: The backbone twists for 1-5 fall within $2^{\circ}$ of each other and the energy differences are within $0.4 \mathrm{kcal} \mathrm{mol}^{-1}$ (less than $k_{B} \mathrm{~T}$ at room temperature).

We hypothesize that non-covalent intramolecular interactions among the side phenyl groups in part lead to the lowest energy, twisted conformation for isolated rubrene derivatives. To assess this point, SAPT0/jun-cc-pVDZ calculations ${ }^{27,31,36,38}$ (simplified throughout the remainder of the discussion as SAPT0) were employed. Here, we thus focus solely on phenyl pairs that are extracted from the tetracene backbone with the dangling bonds on the phenyl groups terminated with hydrogen atoms. This allows us to evaluate the non-covalent interactions among the 5- / 6and 11- / 12-position phenyl pairs (Tables S3 and S4 in the SI).

Before we discuss the energetics of the phenyl-pair interactions in rubrene, it is instructive to recall previous SAPT0 calculations on model, co-facial benzene dimers ${ }^{37}$ with varying electronpoor and electron-rich substituents. At a constrained inter-plane separation of $3.0 \AA$, the very close molecular contacts lead to highly unfavorable exchange-repulsion energies, which average $+46 \mathrm{kcal} \mathrm{mol}^{-1}$ across all dimers considered; this value is nearly twice as large as the stabilizing dispersion terms and three times as large as the stabilizing electrostatic terms (we note that induction only provides a modest degree of stabilization). ${ }^{37}$ The total SAPT0 interaction energy energies (i.e., summation of all four interaction energies) average $+8 \mathrm{kcal} \mathrm{mol}^{-1}$, a result that reveals that the benzene dimers, regardless of the substituent, do not want to lie in such close proximity. Indeed, the minimized (equilibrated) distances for the benzene dimers considered by Hohenstein, Duan, and Sherrill are on the order of $3.7 \AA ;^{37}$ at this distance, the dispersion, 
permanent electrostatic, and induction forces are able to more than counterbalance the repulsive exchange force, leading to overall favorable intermolecular interaction energies (on average -4 kcal $\left.\mathrm{mol}^{-1}\right)$.

Returning to the rubrene structures, the phenyl pairs anchored to the central rings of the tetracene backbone are constrained to an intermolecular separation (based on interatomic distances) of approximately $3 \AA$ (see Figure 2) at their closest point of contact - as with the benzene dimers in Reference ${ }^{37}$. However, to mitigate as much as possible the highly unfavorable exchange interaction at such a close distance, the phenyl rings move away from the co-facial arrangement of a perfect $\mathrm{D}_{2 \mathrm{~h}}$ conformation through two actions: First, the phenyl rings slide by each other to give an angle $\theta_{\mathrm{OP}}$ (defined in Figure 2 ) of 30 to $35^{\circ}$ in the fully minimized structures depending on the functionalization; in the constrained-planar rubrene structures, this motion is limited to 18 to $28^{\circ}$. The phenyl rings also splay out from each other, i.e., the carbon atoms on the exterior of the phenyl rings are further away from each other than those appended to the tetracene backbone, such that the intermolecular separation (by averaging atomic contacts) is $3.5 \AA$.

Therefore, for all phenyl pairs extracted from the rubrene derivatives (Figure 3), the repulsive exchange energy is $c a$. +20 to $+23 \mathrm{kcal} \mathrm{mol}^{-1}$; this energy remains nearly twice as large as the dispersion and three times as large as the electrostatic energies. While these relative values are consistent with those for the benzene dimers at $3 \AA$, they are in absolutes values about half as large as in the constrained $3 \AA$ situation in Reference ${ }^{37}$ due to the average distance in the phenyl pairs being larger than $3 \AA$. In addition, the total intermolecular interaction energies for the phenyl pairs range from $-4.7 \mathrm{kcal} \mathrm{mol}^{-1}$ (i.e., an overall favorable interaction) to $+0.45 \mathrm{kcal} \mathrm{mol}^{-1}$ (i.e., an overall [very small] unstable interaction), depending on the exact nature of the substituents, see Tables S3 and S4. Interactions between phenyl pairs are always more favorable 
in the twisted conformation than in the constrained planar conformation for every derivative considered.

1

2

3

4

5

6

7

8

9

10

11

12

13

14

15

16

17

18

19

20

21

22

23

24

25

26

27

28

29

30

31

32

33

34

35

36

37

38

39

40

41

42

43

44

45

46

47

48

49

50

51

52

53

54

55

56

57

58

59

60 

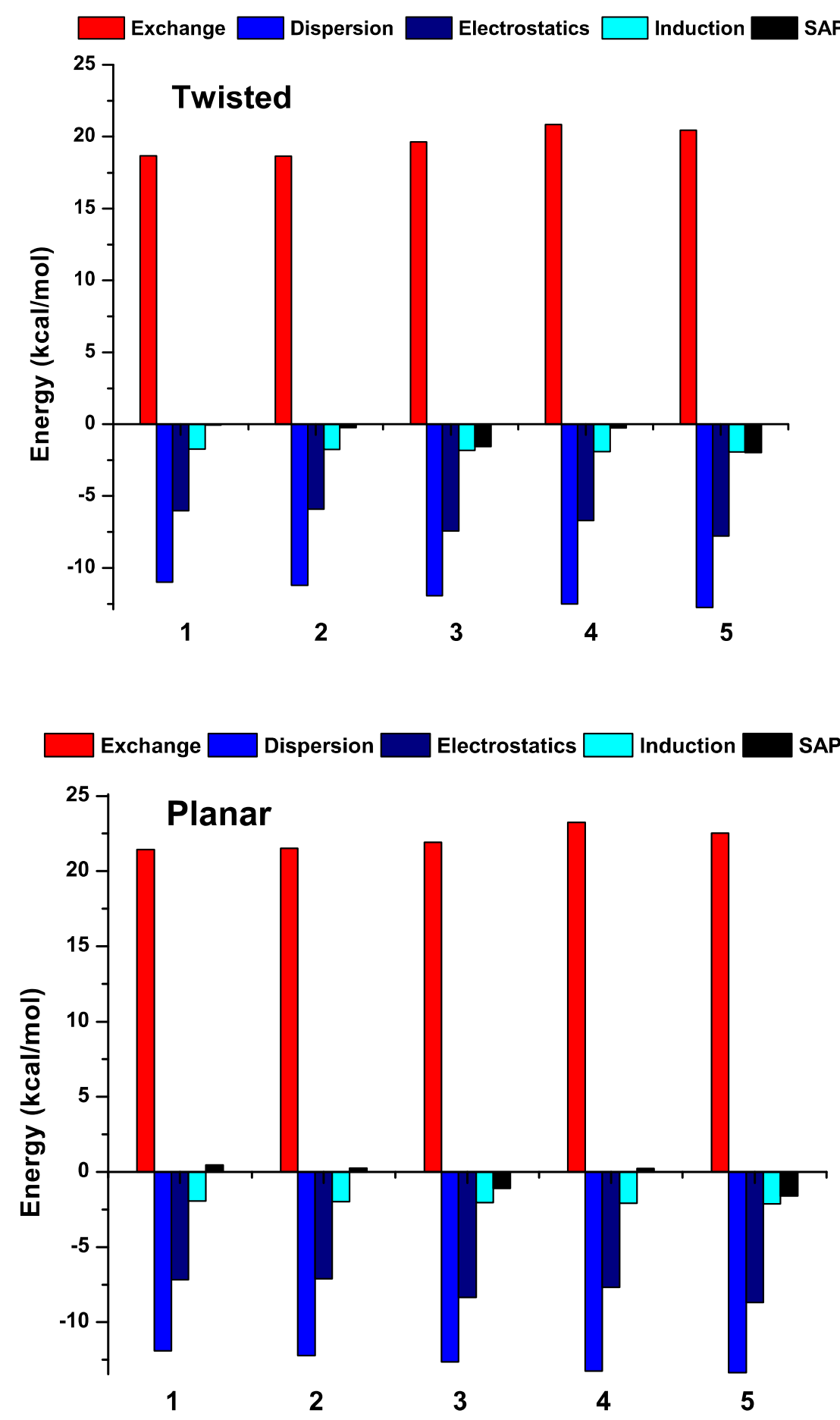

Figure 3. SAPT0/jun-cc-pVDZ computed exchange (red), dispersion (royal blue), electrostatic (navy blue), and induction (light blue) components for phenyl dimers extracted from [top] fullyrelaxed twisted and [bottom] constrained-planar geometries at the IP-tuned $\omega$ B97/cc-pVDZ level of theory. The total SAPT0 interaction energy (black) is also shown. 
We can now understand at least one major reason why isolated rubrene derivatives twist: The phenyl rings contort (slip and splay) themselves to mitigate the repulsive exchange interactions. Even though similar movement is seen for phenyl groups in the constrained-planar and fully relaxed structures, the rubrene twisting remains favorable by $-2 \mathrm{kcal} \mathrm{mol}^{-1}$ as it allows the phenyl rings located on the same side of the tetracene core to slide even further by each other, saving some $-0.5 \mathrm{kcal} \mathrm{mol}^{-1}$ per phenyl pair. Across the rubrene-derivative series, the total SAPT0 intermolecular interaction energies are more stable for the phenyl pairs coming from the fully relaxed, twisted conformations; the main stabilization arises from a reduction of the exchange energy by some $3 \mathrm{kcal} \mathrm{mol}^{-1}$ in the more twisted structures, though the dispersion and electrostatic terms also decrease by $1 \mathrm{kcal} \mathrm{mol}^{-1}$ with increased twisting.

It is interesting to note that the rubrene parent compound $\mathbf{1}$ has the most unfavorable intramolecular phenyl-phenyl interaction energy among the systems studied. This result brings the important point that substitutions can act to stabilize the inter-moiety interactions between the phenyl groups, which is consistent with earlier findings that substituents tend to stabilize $\pi-\pi$ interactions, at least in co-facial arrangements. ${ }^{42}$ In general, substitution with electronwithdrawing groups ( 3 and $\mathbf{5}[\mathbf{7}, \mathbf{8}$, and 12 in the SI]) leads to more favorable interactions among the phenyl pairs as compared to those substituted with electron-donating substituents ( 2 and $\mathbf{4}$ [6, 9, and $\mathbf{1 0}$ in the SI]). Comparing the fluorine-substituted derivatives 11 (tetra[parafluorophenyl]), 12 (bis[perfluorophenly]), and 13 (tetra[perfluorophenyl]), the interaction between the electron-rich phenyl ring and the electron-poor perfluorophenyl ring (in 12) is much more favorable electrostatically than the interaction between two fluorophenyls (11) or two perfluorophenyls (13), contributing to an overall stabilization of the interaction by 2 to $2.5 \mathrm{kcal}$ $\mathrm{mol}^{-1}$ (in either the constrained-planar or twisted geometries). 
To summarize at this stage, the twisted conformations are the most energetically stable for the isolated molecule as they contribute to reduce the repulsive exchange term among the phenyl substituents. It is interesting to note that, for di-phenyl-substituted tetracene backbones, where the substitution is made on the same side (5- and 6-positions, Figure S2 and Table S5) or opposite sides (5- and 12-positions, 5- and 11-positions), the tetracene cores in these systems do not twist. Although a positive SAPT0 interaction energy is derived for the phenyl pairs of the 5,6-di[phenyl], 5,11-di[perfluorophenyl], and 5,12-di[perfluorophenyl] substituted tetracene derivatives, the destabilizing driving force is not large enough to twist the tetracene backbone. Therefore, twisting of the backbone in rubrene derivatives results from the cumulative effect of having two pairs of phenyl groups that need to minimize their exchange repulsion energies, and do so by contorting their conformations.

\section{Solid-State Structures}

We now turn our attention as to why certain rubrene derivatives take on a planar tetracene conformation in the solid state. X-ray crystallography measurements show that the tetracene cores of $\mathbf{1}, \mathbf{3}, \mathbf{5}, \mathbf{7}$, and $\mathbf{1 0}$ (substituted with di-t-butyl groups) are planar in the crystal, while functionalization with methyl groups $(\mathbf{2}, \mathbf{4}$, and 6) or $t$-butyl groups on diagonal arylenes (9) result in a twist of 30 to $40^{\circ}$. SAPT0-based analyses of the side phenyl pairs extracted from the molecular conformations found in the crystals reveal that all of the intra-molecular interactions are globally unfavorable (with the exception of 7, Table S6). Hence, there is no specific stabilization of the intra-molecular phenyl-phenyl interactions in the solid state (compared to the isolated molecules) to assist in planarizing the tetracene core. Importantly, this result points to 
inter-molecular interactions with neighboring molecules in the solid state as the contributing factor.

To address this question, the SAPT0 methodology was used to examine the inter-molecular interactions among the four unique nearest neighbors (see Figure 4 and Tables 1 and S7) of five solid-state planar rubrene derivatives $(\mathbf{1}, \mathbf{3}, \mathbf{5}, \mathbf{7}$, and 10) using their respective crystalline geometries. Starting with the $\pi$-stacked dimer pair (labeled 1-2 in Figure 4) extracted from the $a b$-plane, Table 1 again shows that the destabilizing exchange term is large, a direct consequence of the considerable wavefunction overlap within the dimers (vide infra). However, the presence of significant stabilizing dispersion terms leads to an overall stable interaction energy of -21.36 $\mathrm{kcal} \mathrm{mol}^{-1}$ for $\mathbf{1}$; this stabilization even goes up to $-23.63 \mathrm{kcal} \mathrm{mol}^{-1}$ for both $\mathbf{5}$ and $\mathbf{1 0}$, a result, at least in part, of the increased dispersion coming from the smaller stacking distance.

Table 1: SAPT0/jun-cc-pVDZ comparison of the electrostatic, exchange, induction, and dispersion components of the $\pi$-stacked rubrene dimer pairs (labeled 1-2 in Figure 4) for 1, 3, 5, 7, and 10, as extracted from the crystal structures. Electronic couplings $(t)$ are also provided as determined at the B3LYP/cc-pVDZ level of theory. $\mathrm{D}_{1-2}$ is distance between the planes derived from the tetracene backbones. Dimer interactions for the twisted structures of $\mathbf{2}$ and $\mathbf{4}$ are also given for comparison. All energies in $\mathrm{kcal} \mathrm{mol}^{-1}$ except those between brackets, given in $\mathrm{meV}$.

\begin{tabular}{lccccccc}
\hline \hline System & $\mathrm{D}_{1-2}(\AA)$ & $t$ & Elst. & Ind. & Disp. & Exc. & SAPT0 \\
\hline $\mathbf{1}$ & 3.665 & $2.33[101]$ & -3.24 & -1.39 & -30.78 & +14.14 & -21.36 \\
\hline $\mathbf{3}$ & 3.52 & $2.91[126]$ & -4.38 & -1.49 & -33.59 & +16.52 & -22.94 \\
$\mathbf{5}$ & 3.48 & $3.09[134]$ & -5.00 & -1.60 & -34.95 & +17.93 & -23.63 \\
$\mathbf{7}$ & 3.60 & $2.24[97]$ & -2.34 & -1.13 & -28.18 & +11.94 & -19.71 \\
$\mathbf{1 0}$ & 3.53 & $2.84[123]$ & -4.28 & -1.54 & -34.05 & +16.24 & -23.63 \\
\hline \hline $\mathbf{2}$ & & $0.22[10]$ & -1.14 & -4.27 & -19.16 & +10.03 & -14.55 \\
$\mathbf{4}$ & & $0.21[9]$ & -7.91 & -2.13 & -32.64 & +17.32 & -25.36 \\
\hline \hline
\end{tabular}



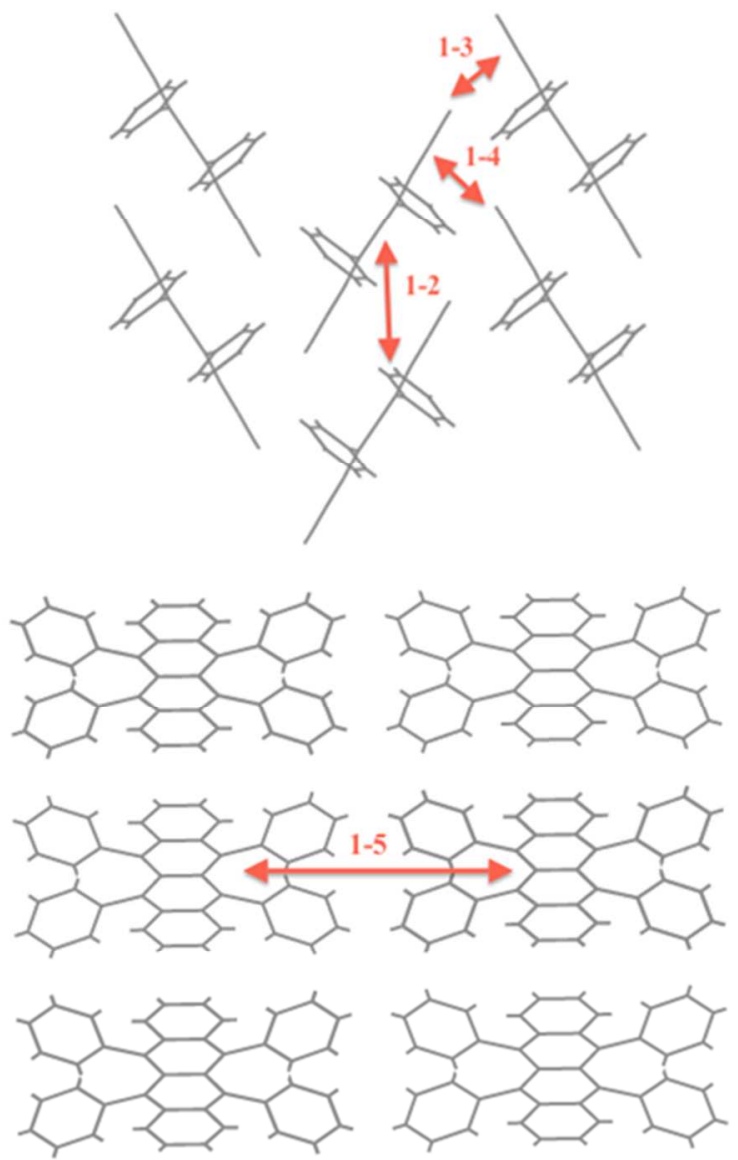

Figure 4. Diagrams of the molecular packing in rubrene (1) along the $\pi$-stacking plane (top) and along the long (b)-axis of the crystal structure (bottom). Dimers, as labeled, were extracted for SAPT0 analysis. All results are reported in Table S7.

An important consequence is that, as the $\pi$-stacking distance decreases and the electronic coupling increases (an established design criterion for organic electronic materials), ${ }^{43}$ the exchange energy very strongly increases due to the enhanced wavefunction overlap. This relationship is readily observed in a model co-facial tetracene dimer (Figure 5, bottom), which points to a major upturn in exchange energy at intermolecular separations ( 3 to $4 \AA$ ) of interest 
for $\pi$-stacked organic materials. Thus, there exists an inherent limit, due to the destabilizing exchange energy, in the maximum electronic couplings achievable in $\pi$-stacked organic semiconductors. The connection between exchange energy and electronic coupling is also observed in the case of the model tetracene dimer when the top molecule is displaced laterally along its long axis; the exchange energy follows a similar oscillatory pattern as the one observed for electronic coupling because of the phase of the frontier $\pi$ orbitals. $^{43}$

When considering materials design, an important aspect is that the destabilizing exchange term can be offset through chemical functionalizations that act to increase the stabilizing components of the non-covalent interactions. This is most evident through a comparison of the $\pi$-stacked intermolecular interactions in $\mathbf{5}$ and $\mathbf{1}$. For $\mathbf{5}$, there is a $21 \%$ increase in the exchange energy (and $25 \%$ increase in the electronic coupling) as compared to 1 , which can be attributed to a smaller backbone-to-backbone stacking distance (3.48 $\AA$ in 5 vs. $3.67 \AA$ in 1); however, there are also a $12 \%$ increase in dispersion, $13 \%$ increase in induction, and $35 \%$ increase in electrostatic energies. Hence, large wavefunction overlap (and electronic coupling) through decreased backbone-to-backbone stacking distances can be obtained by mitigating the necessary accompanying upsurge in exchange repulsion through increasing the dispersion, electrostatic, and induction terms. Fluorination, often used as a means to inductively influence oxidation and reduction potentials, could also be exploited as an effective way to increase the stabilizing noncovalent interactions. The presence of bulky alkyl groups, as in the $t$-butyl groups of $\mathbf{1 0}$, can also be beneficial (when not sterically preventing close contacts) as they can substantially increase dispersion interactions. Analogous functionalization of pentacene by bis(triisopropylsilylethynyl) (TIPS) groups converts the typical herringbone-packing motif of the unsubstituted system to lamellar, $\pi$-stacked structures. ${ }^{44}$ (This is consistent with earlier SAPT0 calculations for pentacene 
at the TIPS-pentacene crystal structure (i.e., without explicit consideration of the TIPS groups), which indeed show a large decrease in the dispersion term). ${ }^{45}$

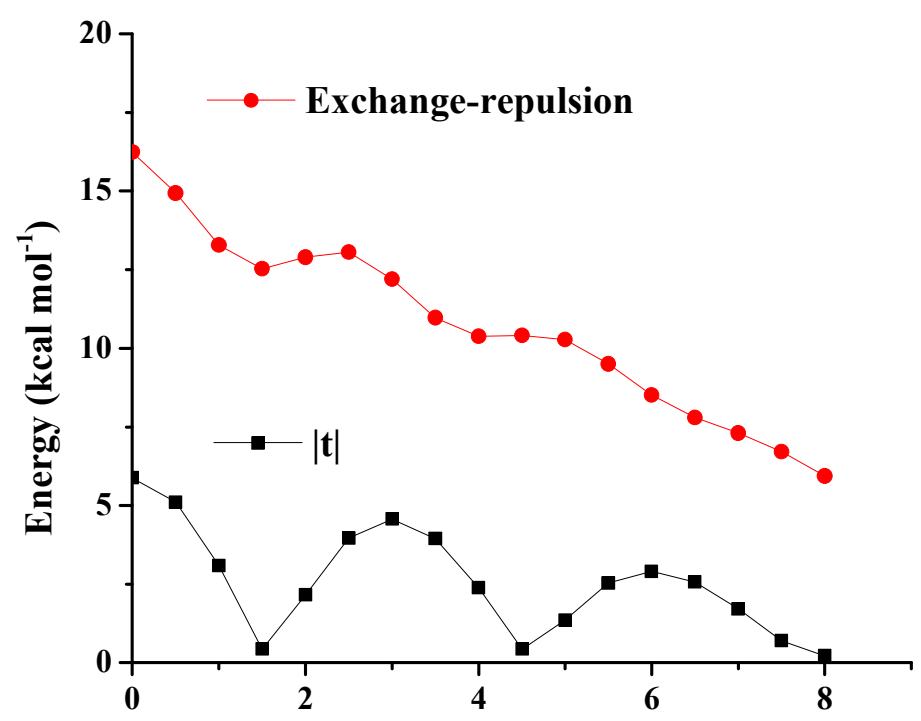

Displacement $(\AA)$

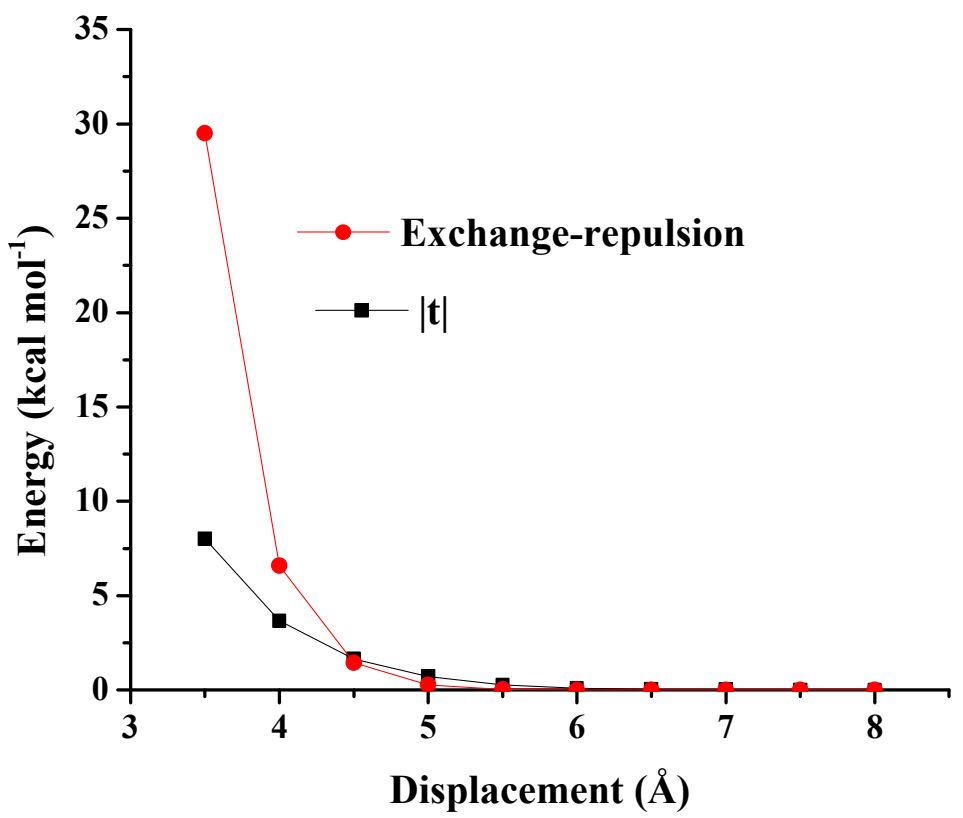

Figure 5. Evolutions of the computed exchange-repulsion energies and HOMO-HOMO electronic couplings $\left(t_{H}\right.$, at the B3LYP/cc-pVDZ level) for a model co-facial dimer of tetracene, as a function of: (bottom) increasing intermolecular separation; and (top) displacement of one of the molecules in the dimer along its long axis. 
While a $\pi$-stacked displaced configuration for a dimer of planar tetracenes is found to be stable, this observation does not explain why it is only certain rubrene derivatives that take on this kind of packing motif, which is prone to display enhanced charge-carrier transport properties. The question thus arises whether inter-molecular interactions with other neighbors aid in planarization of the tetracene core. When we consider the average SAPT0 intermolecular interaction energies for the four nearest molecular neighbors (three molecules within the $a b$ plane and one along the $c$ direction, see Figure 4 and Table S7), a similar degree of stabilization is obtained for each of the planar rubrene structures $(\mathbf{1}, \mathbf{3}, \mathbf{5}, \mathbf{7}$, and 10); chemical substitution can, however, increase the magnitude of these stabilizing interactions compared to $\mathbf{1}$, e.g., $\mathbf{5}$ is 2 kcal $\mathrm{mol}^{-1}$ more stable than $\mathbf{1}$ because of the larger electrostatic and dispersion contributions in the first coordination sphere of the fluorinated complex. These results are consistent with previous observations for a variety of fluorobenzenes ${ }^{46}$ and multi-substituted benzene-benzene dimers. ${ }^{47}$ While certain non-fluorinated rubrene derivatives can also $\pi$-stack $(e . g$., 1 and 10), the impact fluorination has on increasing the stabilizing electrostatic and dispersion interactions can be highlighted through a simple in silico experiment. For instance, see Table S7, starting with the crystal structure for $\mathbf{3}$ and replacing the fluorine atoms with hydrogen atoms (i.e., the molecules of 2 placed in the solid-state packing configuration of 3 ), a decrease of $2 \mathrm{kcal} \mathrm{mol}^{-1}$ is observed in the total inter-molecular interaction energy of the inter-layer rubrene-derivative dimer (dimer 1-5 in Figure 4); this total interaction energy decreases by $3 \mathrm{kcal} \mathrm{mol}^{-1}$ if the $-\mathrm{CF}_{3}$ groups are replaced by hydrogen atoms (i.e., the molecules of $\mathbf{1}$ placed in the solid-state packing configuration of 3). This 1-5 dimer is twice as important as the other nearest-neighbor dimers in determining the lattice energy, because there are four equivalent $1-5$ interactions in the first coordination sphere, compared with only two equivalent 1-2, 1-3, or 1-4 interactions. Overall, 
the interaction energy of 3 in the first coordination sphere is $c a .6 \mathrm{kcal} \mathrm{mol}^{-1}$ more favorable than that of $\mathbf{2}$ in the packing configuration of $\mathbf{3}$ (Table S8).

Interestingly, while the dimer pair in (twisted) $\mathbf{2}$ (see Table 1) represents a stable interaction, the dimer configuration in (twisted) 4 is $c a .2$ to $6 \mathrm{kcal} \mathrm{mol}^{-1}$ more stable when compared to the $\pi$ stacked dimer configurations of the planar crystal structures of $\mathbf{1 , 3}, \mathbf{5}, \mathbf{7}$, and $\mathbf{1 0}$. However, for 2 and 4, the total interaction energies with the four nearest molecular neighbors are 13 to $14 \mathrm{kcal}$ $\mathrm{mol}^{-1}$ for $\mathbf{2}$ and 11 to $12 \mathrm{kcal} \mathrm{mol}^{-1}$ for $\mathbf{4}$ smaller than in $\mathbf{1}, \mathbf{3}$, and $\mathbf{5}$. Therefore, the smaller interaction energies of the $\pi$-stacked rubrene derivatives compared with the twisted dimer 4 are compensated by gains in the intra-layer interactions; the interactions with the three unique intralayer neighbors are considerable (ca. -10 to $-24 \mathrm{kcal} \mathrm{mol}^{-1}$ ) resulting from the close packing (i.e., center-of-mass separation of $<8 \AA$ ); however, for the twisted configurations, there is just a single unique molecular pair with a center-of-mass separation of $\sim 8 \AA$. Inter-layer interactions also play a role in stabilizing the interactions, though to a lesser extent. Intriguingly, even though the interlayer spacing expands due to functionalization on going from $\mathbf{1}(13.41 \AA)$ to $\mathbf{3}(15.59 \AA)$ and $\mathbf{5}$ $(17.07 \AA)$, the interaction energies fall within a small range of 2 to $4 \mathrm{kcal} \mathrm{mol}^{-1}$. Overall, it is the strongly interacting set of close intra-layer neighbors that is identified as the key to $\pi$-stacking of planar structures in rubrene derivatives; if the chemical substitutions reduce these stabilizing interactions, then the unfavorable intra-molecular interactions lead to a twisted rubrene structure, as in $\mathbf{2}$ and $\mathbf{4}$; a packing configuration is then taken in the solid state so as to maximize the interaction energy within at least one dimer pair.

To generalize these results, we investigated the herringbone-packed oligoacene series. In the oligoacenes, both the electronic couplings ( 0.19 to $\left.2.21 \mathrm{kcal} \mathrm{mol}^{-1}\right)$ and exchange-repulsion 
terms $\left(+5.20\right.$ to $\left.+11.98 \mathrm{kcal} \mathrm{mol}^{-1}\right)$ increase from naphthalene to pentacene due to increased wavefunction overlap (Table S9), a critical relationship that was described above. Interestingly, the oligoacenes appear to take on the tight, herringbone packing configuration in order to reduce the impact of the unfavorable exchange energy: For instance, in the case of tetracene, the exchange-repulsion energy is $+9.74 \mathrm{kcal} \mathrm{mol}^{-1}$ for the shortest contact distance in the herringbone crystal structure $(2.90 \AA)$, which can be compared to a considerably larger value of $+28.74 \mathrm{kcal} \mathrm{mol}^{-1}$ for a model co-facial dimer with a separation of $3.50 \AA$. Notably, there is also an increase in the stabilizing dispersion term with increasing acene length - from $-7.35 \mathrm{kcal} \mathrm{mol}^{-1}$ in naphthalene to $-20.50 \mathrm{kcal} \mathrm{mol}^{-1}$ in pentacene - as one might expect simply from the larger number of electrons (and hence the greater ability of the electron density to polarize) as the acene length increases. These results, underlining why the oligoacenes prefer to be arranged in the herringbone packing configuration, suggest that materials chemists interested in designing tight, $\pi$-stacked molecular packing configurations for oligoacene-based molecules need to carefully consider the chemical derivatization necessary to control the non-covalent intermolecular interactions that will lead to such arrangements.

\section{Conclusions}

In this work, we detailed the nature of the non-covalent intra-molecular interactions in rubrene that result in the isolated molecules being twisted. We have then uncovered the type of intermolecular interactions in the solid state that are critical to the formation of the planar, $\pi$-stacked structures, most susceptible to lead to efficient charge-transport properties. 
The comprehensive analysis of these non-covalent interactions allows us to identify (at least some of) the key chemical aspects that can stabilize the planar rubrene conformation and replicate the advantageous packing demonstrated in unsubstituted rubrene. The quantum chemistry-based understanding presented here underlines that improved synthetic derivatization schemes to increase favorable non-covalent interactions, have the potential to improve the materials performance of this benchmark molecular material, in particular with regard to charge transport.

\section{Acknowledgements}

This work at Georgia Tech was supported by the National Science Foundation through the MRSEC Program under Award DMR-0819885, the Chemistry Program under Award CHE1300497, and, for computing resources, the CRIF Program under Award CHE-0946869. CR thanks the University of Kentucky Vice President for Research for start-up funds. JLB acknowledges generous support from King Abdullah University of Science and Technology. The authors thank Dr. Kathryn A. McGarry and Professors C. Daniel Frisbie and Christopher J. Douglas for stimulating discussions.

\section{Supporting Information}

Comparison of computed energy differences for the constrained-planar configurations and the optimized twisted geometries with various functionals; SAPT0/jun-cc-pVDZ values from all 
individual dimers from optimized twisted and constrained planar geometries with $\omega$ B97/ccpVDZ; and SAPT0/jun-cc-pVDZ results for tetracene and di-phenyl substituted tetracene derivatives. This material is available free of charge via the Internet at http://pubs.acs.org.

\section{References}

(1) Kang, J. H.; Filho, D. d. S.; Bredas, J. L.; Zhu, X. Y. Appl. Phys. Lett. 2005, 86, 152115.

(2) Mattheus, C. C.; Dros, A. B.; Baas, J.; Oostergetel, G. T.; Meetsma, A.; de Boer, J. L.; Palstra, T. T. M. Synth. Met. 2003, 138, 475-481.

(3) Jurchescu, O. D.; Mourey, D. A.; Subramanian, S.; Parkin, S. R.; Vogel, B. M.; Anthony, J. E.; Jackson, T. N.; Gundlach, D. J. Phys. Rev. B: Condens. Matter 2009, 80, 085201.

(4) Käfer, D.; El Helou, M.; Gemel, C.; Witte, G. Cryst. Growth Des. 2008, 8, 3053-3057.

(5) Dunitz, J. D.; Gavezzotti, A. Chem. Soc. Rev. 2009, 38, 2622-2633.

(6) Karamertzanis, P. G.; Day, G. M.; Welch, G. W. A.; Kendrick, J.; Leusen, F. J. J.; Neumann, M. A.; Price, S. L. J. Chem. Phys. 2008, 128, 244708.

(7) Price, S. L. Acc. Chem. Res. 2008, 42, 117-126.

(8) Wen, S.; Beran, G. J. O. J. Chem. Theory Comput. 2012, 8, 2698-2705.

(9) Curtis, M. D.; Cao, J.; Kampf, J. W. J. Am. Chem. Soc. 2004, 126, 4318-4328.

(10) Takeya, J.; Yamagishi, M.; Tominari, Y.; Hirahara, R.; Nakazawa, Y.; Nishikawa, T.; Kawase, T.; Shimoda, T.; Ogawa, S. Appl. Phys. Lett. 2007, 90, 102120.

(11) Sutton, C.; Sears, J. S.; Coropceanu, V.; Bredas, J. L. J. Phys. Chem. Lett. 2013, 4, 919924.

(12) In addition to the orthorhombic crystal structure of rubrene, for which large mobilities have been measured, monoclinic and triclinic rubrene polymorphs have been identified; see References 13 and 14. The monoclinic and triclinic polymorphs are not considered in this work for the sake of consistency; the orthorhombic structures considered were grown from vapor-phase deposition procedures, while the polymorphs in References 13 and 14 were developed from solution.

(13) Huang, L.; Liao, Q.; Shi, Q.; Fu, H.; Ma, J.; Yao, J. J. Mater. Chem. 2010, 20, 159-166.

(14) Takeshi, M.; Masashi, Y.; Masahito, U.; Masakazu, Y.; Akiko, N.; Yoshinori, T.; Junichi, T.; Yasuo, K.; Yusuke, M.; Takatomo, S. Jpn. J. Appl. Phys. 2010, 49, 085502.

(15) A previous computational study predicts three of the four crystal structures found experimentally for rubrene, with a triclinic crystal structure predicted to be the minimum configuration. See Reference 16 for further details.

(16) Shigeaki, O.; Toshiaki, M.; Yukihiro, S. Jpn. J. Appl. Phys. 2014, 53, 01 AD02.

(17) Käfer, D.; Ruppel, L.; Witte, G.; Wöll, C. Phys. Rev. Lett. 2005, 95, 166602.

(18) Petrenko, T.; Krylova, O.; Neese, F.; Sokolowski, M. New J. Phys. 2009, 11, 015001.

(19) Kytka, M.; Gisslen, L.; Gerlach, A.; Heinemeyer, U.; KovaC, J.; Scholz, R.; Schreiber, F. J. Chem. Phys. 2009, 130, 214507. 
(20) Casanova, D. J. Chem. Theory Comput. 2013, 10, 324-334.

(21) Paraskar, A. S.; Reddy, A. R.; Patra, A.; Wijsboom, Y. H.; Gidron, O.; Shimon, L. J. W.; Leitus, G.; Bendikov, M. Chem. Eur. J. 2008, 14, 10639-10647.

(22) Duhm, S.; Xin, Q.; Hosoumi, S.; Fukagawa, H.; Sato, K.; Ueno, N.; Kera, S. Adv. Mater. 2012, 24, 901-905.

(23) Jurchescu, O. D.; Meetsma, A.; Palstra, T. T. M. Acta Crystallogr., Sect. B: Struct. Sci 2006, 62, 330-334.

(24) McGarry, K. A.; Xie, W.; Sutton, C.; Risko, C.; Wu, Y.; Young, V. G.; Bredas, J. L.; Frisbie, C. D.; Douglas, C. J. Chem. Mater. 2013, 25, 2254-2263.

(25) Haas, S.; Stassen, A. F.; Schuck, G.; Pernstich, K. P.; Gundlach, D. J.; Batlogg, B.; Berens, U.; Kirner, H. J. Phys. Rev. B: Condens. Matter 2007, 76, 115203.

(26) Bergantin, S.; Moret, M. Cryst. Growth Des. 2012, 12, 6035-6041.

(27) Jeziorski, B.; Moszynski, R.; Szalewicz, K. Chem. Rev. 1994, 94, 1887-1930.

(28) Burns, L. A.; Mayagoitia, A. V.; Sumpter, B. G.; Sherrill, C. D. J. Chem. Phys. 2011, 134, 084107.

(29) Chai, J.-D.; Head-Gordon, M. J. Chem. Phys. 2008, 128, 084106.

(30) Zhao, Y.; Truhlar, D. G. Theor. Chem. Acc. 2008, 120, 215-241.

(31) R. A Kendall, T. H. D., Jr., R. J. Harrison J. Chem. Phys. 1992, 96, 6796-6806.

(32) Senthilkumar, K.; Grozema, F. C.; Bickelhaupt, F. M.; Siebbeles, L. D. A. J. Chem. Phys. 2003, 119, 9809-9817.

(33) Valeev, E. F.; Coropceanu, V.; da Silva Filho, D. A.; Salman, S.; Bredas, J. L. J. Am. Chem. Soc. 2006, 128, 9882-9886.

(34) Baer, R.; Livshits, E.; Salzner, U. Annu. Rev. Phys. Chem. 2010, 61, 85-109.

(35) Hohenstein, E. G.; Sherrill, C. D. J. Chem. Phys. 2010, 132, 184111-184110.

(36) Hohenstein, E. G.; Sherrill, C. D. J. Chem. Phys. 2010, 133, 104107.

(37) Hohenstein, E. G.; Duan, J.; Sherrill, C. D. J. Am. Chem. Soc. 2011, 133, 13244-13247.

(38) Papajak, E.; Zheng, J.; Xu, X.; Leverentz, H. R.; Truhlar, D. G. J. Chem. Theory Comput. 2011, 7, 3027-3034.

(39) Jurečka, P.; Černý, J.; Hobza, P.; Salahub, D. R. J. Comput. Chem. 2007, 28, 555-569.

(40) Marshall, M. S.; Burns, L. A.; Sherrill, C. D. J. Chem. Phys. 2011, 135, 194102.

(41) Norton, J. E.; Houk, K. N. J. Am. Chem. Soc. 2005, 127, 4162-4163.

(42) Sinnokrot, M. O.; Sherrill, C. D. J. Am. Chem. Soc. 2004, 126, 7690-7697.

(43) Bredas, J. L.; Calbert, J. P.; da Silva Filho, D. A.; Cornil, J. Proc. Natl. Acad. Sci. U.S.A. 2002, 99, 5804-5809.

(44) Anthony, J. E. Chem. Rev. 2006, 106, 5028-5048.

(45) Ryno, S. M.; Risko, C.; Bredas, J.-L. J. Am. Chem. Soc. 2014, 136, 6421-6427.

(46) Thalladi, V. R.; Weiss, H.-C.; Bläser, D.; Boese, R.; Nangia, A.; Desiraju, G. R. J. Am. Chem. Soc. 1998, 120, 8702-8710.

(47) Ringer, A. L.; Sinnokrot, M. O.; Lively, R. P.; Sherrill, C. D. Chem. Eur. J. 2006, 12, 3821-3828. 


\section{TOC Graphic}

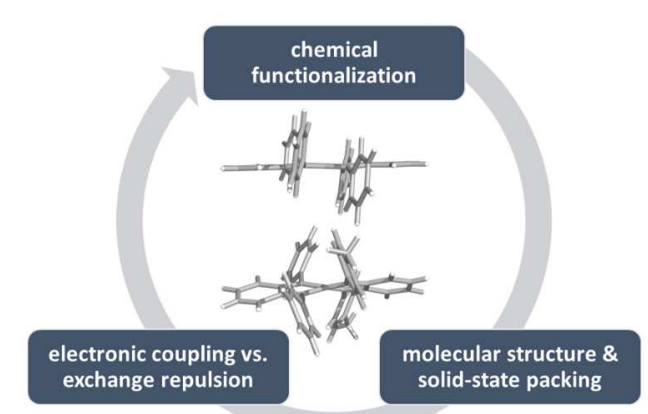

17

18

19

20

21

22

23

24

25

26

27

28

29

30

31

32

33

34

35

36

37

38

39

40

41

42

43

44

45

46

47

48

49

50

51

52

53

54

55

56

57

58

59

60

ACS Paragon P1ys Environment 